\title{
Hotelling revisits the lab: equilibration in continuous and discrete time
}

\author{
Curtis Kephart ${ }^{1} \cdot$ Daniel Friedman $^{1}$
}

Received: 12 October 2014/Accepted: 10 April 2015/Published online: 25 April 2015

(C) Economic Science Association 2015

\begin{abstract}
We investigate experimentally the impact of continuous time on a fourplayer Hotelling location game. The static pure strategy Nash equilibrium (NE) consists of firms paired-up at the first and third quartiles of the linear city. In a repeated simultaneous move (discrete time) treatment, we largely replicate previous findings in which subjects fail to converge to the NE. However, in asynchronous move (continuous time) treatments we see clear convergence towards the NE.
\end{abstract}

Keywords Hotelling model - Laboratory experiment - Asynchronous move

JEL Classification $\quad$ C73 $\cdot$ C92 $\cdot$ D21 $\cdot$ D43

\section{Introduction}

In his seminal model of spatial competition, Hotelling (1929) analyzed the behavior of two sellers of a homogenous product choosing price and location in a bounded, one-dimensional marketplace. The model has since been expanded to allow numerous sellers to interact strategically in more general marketplaces. As the preeminent model of spatial competition, it has been widely applied, e.g., in

Electronic supplementary material The online version of this article (doi:10.1007/s40881-015-0009-z) contains supplementary material, which is available to authorized users.

Curtis Kephart

curtiskephart@gmail.com

Daniel Friedman

dan@ucsc.edu

1 Economics Department, University of California, Santa Cruz, 1156 High Street, Santa Cruz, CA 95064, USA 
industrial organization to analyze geographic competition and product differentiation (Netz and Taylor 2002), and in political economy as a tool to analyze voting dynamics (Downs 1957).

Here we investigate the dynamic foundations of this static model. How does the ability to quickly adjust product characteristics, and quickly respond to competitors' repositioning, affect firm behavior? The question is theoretical but quite relevant to $21 \mathrm{st}$ century applications as firms adopt new technology and new management practices that encourage them to compete on agility and positioning. Product cycles (and rebranding) seem to be accelerating in consumer electronics and lifestyle goods, while in other industries like enterprise software - for example in web-base applications like those for analytics and customer relationship management—permit their makers to continually tweak their product and respond to competitor repositioning.

Outside of voter analysis, empirical tests of the Hotelling model have been sparse. For differentiated products, for example, there is often no consensus on how best to define the attribute space, and most firms have been understandably reluctant to allow access to their data.

Experimental methods offer an empirical approach that avoids these problems, but the results so far have been mixed at best. Brown-Kruse et al. (1993) and Brown Kruse and Schenk (2000) investigate a duopoly model with varying customer densities over a finite one-dimensional action space. Collins and Sherstyuk (2000) look into a three-agent model with inelastic demand and uniform prices. There exists no pure-strategy equilibrium in this set-up (Eaton and Lipsey 1975). Shaked (1982) finds a unique mixed strategy equilibrium in which players randomize uniformly over the second and third quartiles. Collins and Sherstyuk (2000) find little support for Shaked's equilibrium hypothesis.

In work most closely related to this paper, Huck et al. (2002) investigate the fourplayer implementation of the location-only model. Eaton and Lipsey show that all Nash equilibria are in pure strategies with two players located back-to-back at the first quartile, and the remaining two players similarly located at the third quartile. Like Collins and Sherstyuk, Huck et al. find that the empirical distribution of locations is quite different than this NE distribution-subjects exhibit a "Wshaped" distribution of locations, with significant clustering near the second quartile (the median) as well as near the first and third quartile.

Using novel software for conducting economic experiments, we investigate the fourplayer location-only model, uniform customer density, inelastic demand and a bounded, finite action space. We compare a discrete time treatment with two continuous time treatments in which subjects are able to adjust their locations either with (or else without) a speed limit. We replicate earlier results that human subjects generally fail to converge to the distinctive equilibrium in discrete time, but establish for the first time that they do converge reliably to the equilibrium in both continuous time treatments.

\section{Hotelling location model}

Each firm $i=1,2, \ldots, n$ chooses a location $s_{i} \in[0,1]$. Firms produce homogenous goods with identical mill prices and linear transport costs. Each of a uniform 
continuum of consumers inelastically purchase a single unit at the lowest delivered price, i.e., from the closest firm.

Payoffs are determined as follows. Sort the strategy profile $\left(s_{1}, s_{2}, \ldots, s_{n}\right)$, so $S_{[1]}=\min \left\{s_{1}, s_{2}, \ldots, s_{n}\right\}, \quad S_{[2]} \quad$ is the second lowest location, $\ldots, \quad$ and $S_{[n]}=\max \left\{s_{1}, s_{2}, \ldots, s_{n}\right\}$, so $S_{[1]} \leq S_{[2]} \leq \cdots \leq S_{[n]}$. If there are exact ties $\left(s_{i}=s_{j}\right)$, then average the payoffs defined below over all feasible assignments of the tied firms.

Normalizing unit profit to 1.0 , firm $i$ 's payoff is the length of its territory. As illustrated in Fig. 1, that territory (except for the 'edge' firms [1] and $[n]$ ) extends from the midpoint of the interval $\left[S_{[i-1]}, S_{[i]}\right]$ with the firm just below to the midpoint of the interval $\left[S_{[i]}, S_{[i+1]}\right]$ with the firm just above. Thus the payoff function is

$$
\Pi_{i}=\frac{1}{2}\left(S_{[i+1]}-S_{[i-1]}\right), \quad i=2, \ldots, n-1
$$

with

$$
\begin{aligned}
& \Pi_{1}=S_{[1]}+\frac{1}{2}\left(S_{[2]}-S_{[1]}\right) \\
& \Pi_{n}=\left(1-S_{[n]}\right)+\frac{1}{2}\left(S_{[n]}-S_{[n-1]}\right) .
\end{aligned}
$$

Equilibria in this game are sensitive to the number $n$ of competing firms. It is well known that for $n=4$ there is a unique pure NE.

Proposition 1 The Hotelling location game for $n=4$ players has a unique pure Nash equilibrium, up to relabeling of players. The unique sorted equilibrium profile is $S_{[1]}=S_{[2]}=\frac{1}{4}$ and $S_{[3]}=S_{[4]}=\frac{3}{4}$.

That is, in NE players are paired "back-to-back" at the first and third quartiles. It is easy to check that this is indeed a NE: by Eq. (2) a deviation to $[0,0.25)$ or to $(0.75,1]$ clearly shrinks the deviator's territory and payoff, while by (1) a deviation to $(0.25,0.75)$ shifts the deviator's territory but does not increase payoff. For a formal proof, see appendix B of Huck et al. (2002), and for a proof of the uniqueness of pure NE, see Eaton and Lipsey (1975).

\subsection{Dynamic considerations}

In previous laboratory examinations of the Hotelling location-only game (Collins and Sherstyuk 2000; Huck et al. 2002), subjects were given random initial positions and allowed to select new actions simultaneously in discrete time, i.e., in a finitely repeated game. Although the stage game is symmetric and constant sum (the total payoff is always 1.0), the players face considerable strategic uncertainty-to chose well, they must accurately predict their opponents' next location choices. The difficulty of predicting increases considerably as the number of players increases beyond $n=2$. Players may also face a coordination issue; namely, where should 


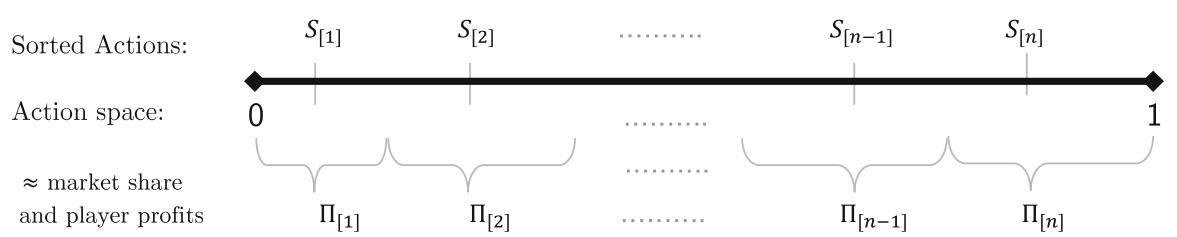

Fig. 1 Hotelling Linear City action space

each player go in the NE configuration? Thus it is easy to question the relevance of the static pure Nash equilibrium to a game with this type of dynamic structure.

To help understand what we might see in the dynamic game we ran computer simulations. In a discrete location space with 101 grid points and random initial locations, our automated agents played their myopic best response: in period $t+1$ each player allowed to move chooses a location that would maximize payoff given the period $t$ location profile of the other players. Of more than 1000 simulations each of 600 periods in which agents moved simultaneously (everyone allowed to move every period), none $(0 \%)$ converged to the NE and most locations were in the vicinity of the midpoint. (We tried several variants, but achieved convergence to $\mathrm{NE}$ in simultaneous move simulations only when initial locations were quite close to the $\mathrm{NE}$ and moves were limited to small increments). By contrast, in more than 1000 asynchronous simulations in which players move one at a time in fixed order, all $(100 \%)$ converged to NE, most of them within 200 periods and from a diverse set of initial locations. See the Online Supplementary Materials for more details.

These simulations suggest that the static model's predictive power may hinge on the dynamic specifications. Moves are almost always asynchronous in continuous time, so in that respect it is similar to our turn-based simulations.

\subsection{Testable predictions}

Our human subject experiment is designed to test two hypotheses suggested by the preceding theoretical discussion.

Hypothesis 1 Observed average deviations from the static Nash equilibrium (NE) will decrease over time in all treatments.

Hypothesis 2 Smaller average deviations from NE will be observed in continuous time (asynchronous) treatments than in the discrete time (synchronous) treatment.

\section{Experiment}

The experiment was programmed in ConG, software designed to implement continuous time economics experiments (Pettit et al. 2013). Subjects choose their target location using their mouse to click or to drag a slider, the black rectangle seen at the bottom of the screen in Fig. 2. Subjects may also use the left and right arrow keys to shift locations incrementally. The horizontal position of the large green dot (currently with a score of 63.6) and black rectangle indicates the subject's current 


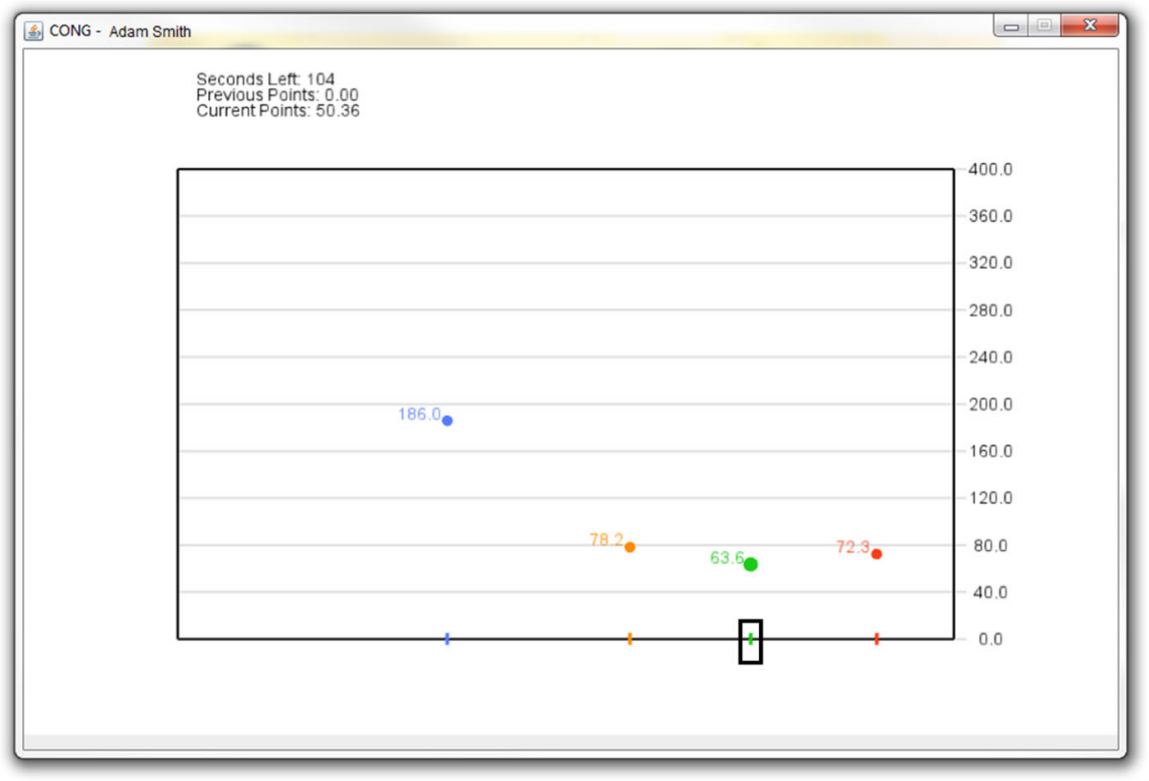

Fig. 2 Hotelling game interface for continuous treatments

location, and other players' current locations are indicated with smaller dots of different colors. The vertical height of each dot indicates the players' current flow payoffs, also indicated by the number displayed by each players' dot. Accumulated flow payoffs are shown in the "Current Points" field, while points earned in all previous paid periods are indicated in the "Previous Points" field.

\subsection{Treatments}

We study three main treatments. The first is discrete time ("Discrete"). Periods are divided into $n$ equal-length subperiods. Within each subperiod, subjects are freely able to move their location target using the mouse or arrow keys, but subperiod payoffs are determined solely by the target location profile chosen at the very end of the subperiod. Only at that point do subjects see the other players' chosen locations. Subjects see a progress bar filling smoothly to indicate when the subperiod will end, and a "Subperiods Left" field counts down until the end of the period (neither are visible in the continuous time UI shown in Fig. 2). Payoffs for the entire period are the integral across subperiods of the piecewise constant flow payoffs, or equivalently, the average of the lump sum subperiod payoffs. A video of this and the other two treatments may be seen online at http://youtu.be/NX6L1mV9iII.

The other two treatments are continuous time. In continuous-time slow ("Slow") when subjects select a new target location their current position moves toward the target at a constant rate (the "speed limit"). The chosen speed limit is such that it would take $30 \mathrm{~s}$ to traverse the entire interval. 
In continuous-time instant ("Instant"), the subject's current position moves immediately to the chosen target, with no perceptible delay. Actual latencies in our lab are less than $50 \mathrm{~ms}$; that is, no more than $50 \mathrm{~ms}$ elapse from the time a subject clicks a new location until the time when the effect of that click is revealed on all subjects' displays. Of course, human reaction times are considerably longer than that, and subjects perceive the action as continuous in this treatment.

\subsection{Procedures}

Treatments are varied across sessions. Subjects are matched and rematched within "silos" of six subjects, and most sessions involve two silos. Sessions run for twelve periods, each period lasting three minutes. Discrete time periods are broken into 60 subperiods of $3 \mathrm{~s}$ each.

As shown in Table 1, each period a different subset of the six subjects in a silo play the $n=4$ location game, while the two excluded subjects each play in a separate four-player game against three automated agents ("robots"). Robots reset their target position every $30 \mathrm{~s}$ following a specified sequence. For example subjects matched with robots in periods 1 through 3 face algorithm R1. The robot algorithms are held constant within each three-period set (periods 1-3, 4-6, 7-9 and 10-12). Subjects were told ahead of time that in one-third of periods their counterpart players would be players controlled by an automated computer program, and each subject faced each sequence once. ${ }^{1}$

Within each time treatment, each subject has equivalent opportunities to profit in robot periods, and so profit differences measure individual subjects' relative ability to best respond. However, opportunities differ across time treatments- e.g., robots in Instant are sitting ducks for $30 \mathrm{~s}$ at a time while in Slow they often are moving steadily and the human subject is also constrained by the speed limit-so profit comparisons are less meaningful across treatments.

Our matching procedure balances several considerations. By the "Folk Theorem", a vast number of location configurations are supported in equilibrium with infinite horizon fixed matchings, and even with a finite horizon one might see fairly arbitrary conventions emerge as epsilon equilibria. Random matchings seem better suited to test static non-cooperative game predictions, but randomizing over large subject pools over many periods has two drawbacks. It produces relatively few "independent" observations, and enables a few confused subjects to "contaminate" a large fraction of the data. ${ }^{2}$ Our solution was to match a different set of four subjects each period within a relatively small silo. Each silo gives us an independent

\footnotetext{
${ }^{1}$ The location sequences for each robot group are as follows, R1: [0.25, 0.5,0.75], [0.12,0.33,0.55], [0.23, $0.76,0.85],[0.35,0.59,0.64],[0.25,0.26,0.75],[0.23,0.75,0.76],[0.45,0.5,0.8]$. R2: $[0.2,0.3,0.58]$, $[0.29,0.59,0.6],[0.55,0.75,0.85],[0.25,0.26,0.74],[0.29,0.35,0.75],[0.23,0.75,0.76],[0.33,0.5,0.66]$. R3: $[0.75,0.5,0.25], \quad[0.88,0.67,0.45], \quad[0.77,0.24,0.15], \quad[0.65,0.41,0.36], \quad[0.75,0.74,0.25]$, [0.77, $0.25,0.24],[0.55,0.5,0.2]$. R4: [0.8,0.7,0.42], [0.71,0.41,0.4], [0.45,0.25,0.15], [0.75,0.74,0.26], [0.71,0.65,0.25], [0.77, $0.25,0.24],[0.67,0.5,0.34]$.

${ }^{2}$ In pilot sessions, post-experiment surveys and relatively low earnings for a few subjects indicated persistent confusion. Several other players exposed to one of these confused individuals exhibited less systematic behavior later in the session, even when no longer playing against that individual.
} 
Table 1 Matching protocol

\begin{tabular}{|c|c|c|c|c|c|c|c|c|c|c|c|c|}
\hline \multirow[t]{2}{*}{ Subject } & \multicolumn{12}{|c|}{ Period } \\
\hline & 1 & 2 & 3 & 4 & 5 & 6 & 7 & 8 & 9 & 10 & 11 & 12 \\
\hline 1 & $\mathrm{R} 1$ & $4 p$ & $4 p$ & $4 p$ & $4 p$ & $\mathrm{R} 2$ & $4 p$ & $4 p$ & R3 & $4 p$ & $4 p$ & $\mathrm{R} 4$ \\
\hline 2 & $\mathrm{R} 1$ & $4 p$ & $4 p$ & $\mathrm{R} 2$ & $4 p$ & $4 p$ & $4 p$ & $\mathrm{R} 3$ & $4 p$ & $4 p$ & $\mathrm{R} 4$ & $4 p$ \\
\hline 3 & $4 p$ & $\mathrm{R} 1$ & $4 p$ & $4 p$ & $\mathrm{R} 2$ & $4 p$ & $4 p$ & $4 p$ & R3 & $4 p$ & R4 & $4 p$ \\
\hline 4 & $4 p$ & $\mathrm{R} 1$ & $4 p$ & $\mathrm{R} 2$ & $4 p$ & $4 p$ & $\mathrm{R} 3$ & $4 p$ & $4 p$ & $\mathrm{R} 4$ & $4 p$ & $4 p$ \\
\hline 5 & $4 p$ & $4 p$ & $\mathrm{R} 1$ & $4 p$ & $\mathrm{R} 2$ & $4 p$ & $\mathrm{R} 3$ & $4 p$ & $4 p$ & $4 p$ & $4 p$ & R4 \\
\hline 6 & $4 p$ & $4 p$ & $\mathrm{R} 1$ & $4 p$ & $4 p$ & $\mathrm{R} 2$ & $4 p$ & $\mathrm{R} 3$ & $4 p$ & $\mathrm{R} 4$ & $4 p$ & $4 p$ \\
\hline
\end{tabular}

Player assignments are shown for each 6-player silo. The four subjects labelled " $4 \mathrm{p}$ " in each column play the Hotelling location game that period; the other two subjects each play against three automated agents ("robots") using one of four algorithms (R1-R4) described in the text

observation, and confines possible contamination. As a bonus, the extra two subjects in each silo each period enabled individual performance comparisons against robots.

Sessions were conducted in the LEEPS laboratory at the University of California, Santa Cruz in February and March 2013. A total of 54 human subjects (randomly assigned into 9 silos) were drawn from the LEEPS Lab subject pool using recruitment package ORSEE (Greiner 2004). Sessions lasted about 90 minutes, starting with instructions (reproduced in the Online Supplementary Materials), two practice periods and a quiz, followed by the 12 periods specified in Table 1, and finally cash payment. Subjects were told that they would be matched with three robots (algorithms unspecified, but subjects found it obvious that they were not human) in one-third of the periods, and with three other human participants in the room (silos unspecified, and their existence was not obvious to subjects) in the other two-thirds. Sessions also included a standard Holt-Laury risk preference test, but the elicited values turned out to provide negligible explanatory power and, to conserve space, are not discussed further.

Our location game is constant sum, and points were scaled so that each period the total score of all four players summed to 400 or 100 points per-player per-period on average. Subjects received a \$5 show-up fee and between $\$ 0.025$ and $\$ 0.034$ for each point earned over the entirety over the session in excess of 1000 points, including both all-human periods and robot periods.

Participating subjects received an average total payment of \$14.22.

\section{Results}

The three panels of Fig. 3 show the overall distribution of locations by treatment in the last half of each session (periods 7-12). In the first panel for the Discrete treatment, there is little evidence of the NE configuration. There are modest modes near the NE quartile points 0.25 and 0.75 , but also a mode near the non-NE quartile (median) point 0.50 , and a noticeable aversion to edge locations. The data of Huck 

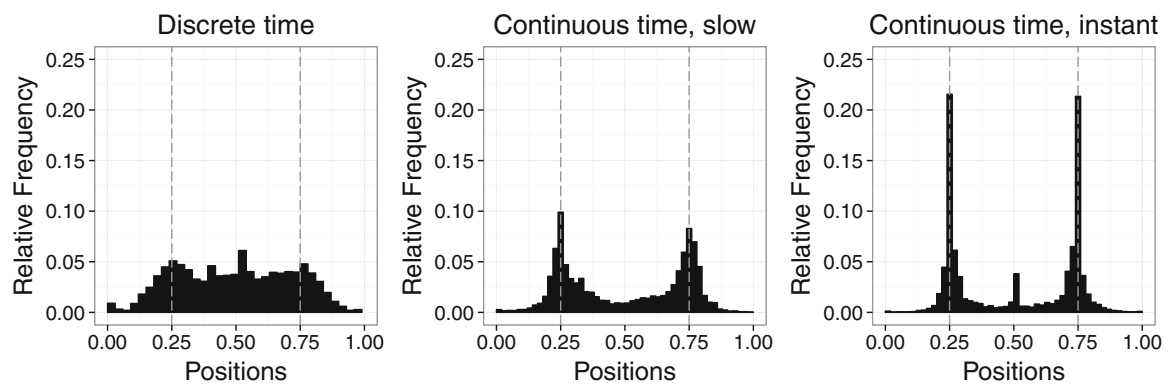

Fig. 3 Relative frequency distribution of player locations, by treatment. Note: Location distributions are from the later half of sessions, excluding any period involving robots

et al. (2002) has similar properties, but all three modes are sharper and location choices between the modes are less common than in our data. The second panel from the Slow treatment shows that we have strong modes in the vicinity of both NE quartile points, and no mode at 0.5 ; indeed, choices distant from 0.25 and 0.75 are rare and transient. The third Instant treatment panel shows sharp modes at the NE quartile points, and rather little activity elsewhere. (The small mode at 0.5 mainly reflects the behavior of a single player who stubbornly occupied that position for a number of periods in one session, despite unimpressive payoffs).

The histograms in Fig. 3 suggest better convergence to NE in Instant than in Slow, and little or no convergence in Discrete. To test our hypotheses more formally, we define a metric for the average absolute distance from NE, as follows. Sort the players' time- $t$ locations as usual: $S_{[1] t}$ is the location of the "left most" player at time $t, S_{[2] t}$ is second from the left, etc., so that $S_{[1] t} \leq S_{[2] t} \leq S_{[3] t} \leq S_{[4] t}$. Then AvgAbsDist at time or subperiod $t$ is:

$$
\text { AvgAbsDist }_{t}=\frac{1}{4}\left(\left|S_{[1] t}-0.25\right|+\left|S_{[2] t}-0.25\right|+\left|S_{[3] t}-0.75\right|+\left|S_{[4] t}-0.75\right|\right)
$$

Clearly $A v g A b s D i s t=0$ at a NE profile, and is bounded above by 0.5 (achieved when all $S_{[i] t}=0$ or all =1). Ten million Monte Carlo simulations indicated that its expected value for a sample drawn from a uniform random distribution is 0.1715 , with a median of 0.1646 .

The overall median value of AvgAbsDist in our Discrete data is 0.1417 , not much different from the random benchmark of 0.1646, while the median values in Slow and Instant, 0.0639 and 0.0461 respectively, are substantially lower. However, looking at the overall average AvgAbsDist of each session period we can reject the null hypothesis that the median is 0 in each treatment.

Is there a trend towards NE across periods? Figure 4 plots AvgAbsDist in humanonly location games averaged period-by-period over all silos in each treatment. In the two Continuous treatments we do see a trend towards 0 (i.e., towards NE) after the first few periods, but it seems to level off thereafter. In Discrete periods, AvgAbsDist is not much below the random benchmark (the gray dashed line) and has no noticeable downward trend. 


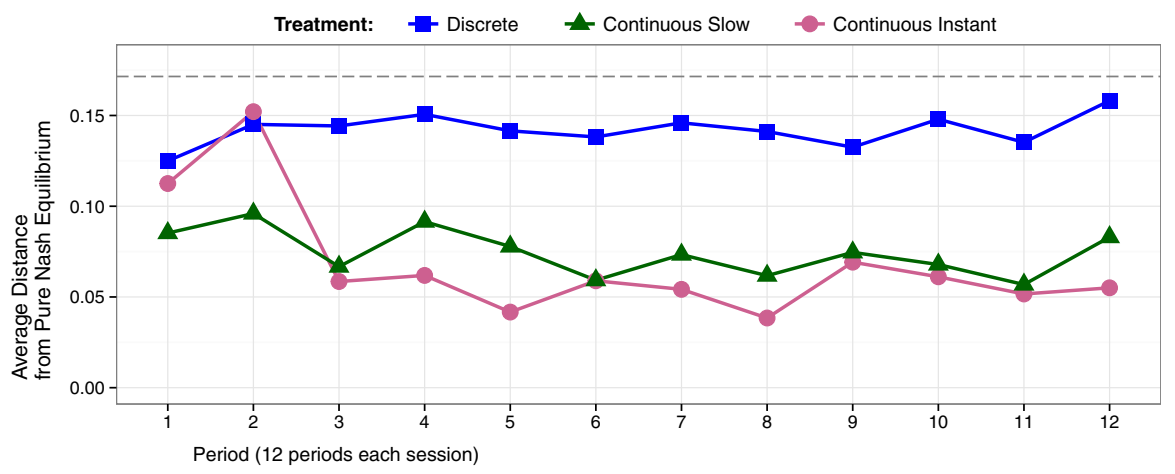

Fig. 4 AvgAbsDist by period and treatment

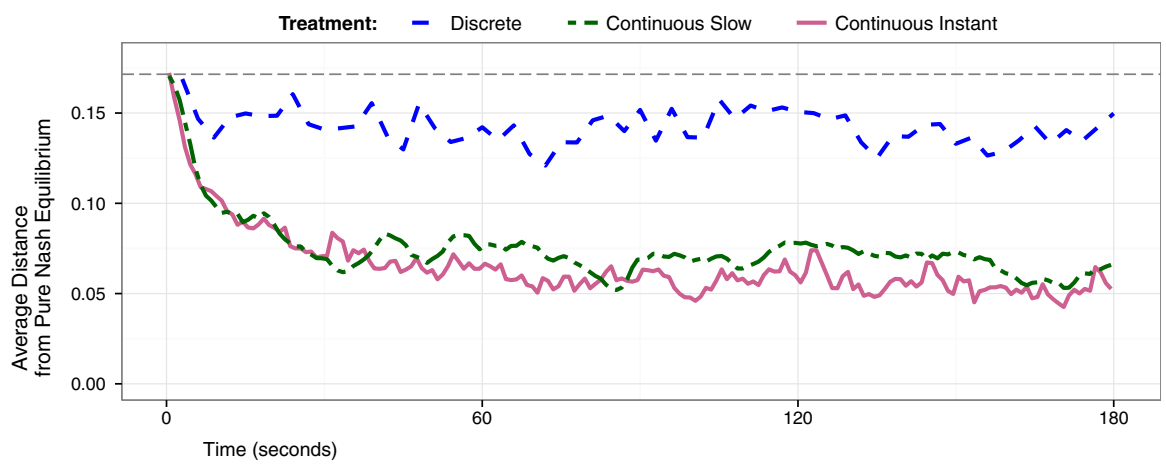

Fig. 5 AvgAbsDist by second, by treatment over the period

Are there trends within a typical period? Figure 5 plots AvgAbsDist in humanonly location games averaged second-by-second (or, for Discrete, subperiod-bysubperiod) over all periods in each treatment. The software initializes subjects at uniform random locations, thus at time zero AvgAbsDist is about 0.17, as expected. Again, in the Discrete treatment, there is little trend towards NE within the period, while in the continuous treatments there is a clear trend at first but it stagnates after about $40 \mathrm{~s}$.

Our main hypothesis tests are based on the following variant of the regression model of Noussair et al. (1995), applied to human-only data from latter half of all sessions (periods 7 through 12):

$$
A v g A b s \text { Dist }_{j t}=\beta_{1}\left(\frac{1}{t}\right)+\beta_{21} \cdot D_{1}\left(\frac{t-1}{t}\right)+\beta_{22} \cdot D_{2}\left(\frac{t-1}{t}\right)+\beta_{23} \cdot D_{3}\left(\frac{t-1}{t}\right)+u_{j t}
$$

where $A v g A b s$ Dist $_{j t}$ is the observed average absolute distance from the pure Nash equilibrium configuration, in treatment $j$ at time $t$-each $100 \mathrm{~ms}$ in continuous time or subperiod $t$ in the case of discrete time. $D_{1}$ is a dummy variable that is equal to 1 if the observation is from treatment $j=1$, Discrete time. Likewise the dummies $D_{2}$ 
and $D_{3}$ are for observations from the Slow and Instant time treatments, respectively. Finally, $\mathrm{u}$ is the random error that is normally distributed with mean zero.

The model offers an estimate of the direction of convergence. The $\beta_{1}$ term has the interpretation as the $y$-axis intercept, i.e. where the time series starts at time $t=1$. The coefficient $\beta_{2 j}$ can be interpreted as the value AvgAvgDist ${ }_{j t}$ converges toward as $t \rightarrow \infty$ in treatment $j$, since $\frac{t-1}{t} \rightarrow 1$ as $t \rightarrow \infty$. Equation (4) imposes a single origin coefficient, and imposes equal convergence target coefficients $\beta_{2 j}$ across silos and periods, but allows those targets to vary across treatments.

Estimates are shown in Table 2. Since we observe repeated interactions with individuals in the same silo, we cluster errors at the silo level and report standard errors corrected first-order autocorrelation using the Newey-West (1986) method for panel data sets.

Result 1 Locations in the Discrete time treatment remain far away from the Nash equilibrium profile.

Support for Result 1 Coefficient estimate $\widehat{\beta}_{21}=0.140$ in Table 2 is statistically and "economically" very different from zero. There is a weak tendency for the distance from NE to decrease over time, inasmuch as $\widehat{\beta}_{21}$ is less than the estimate

Table 2 Estimates of Eq. (4)

\begin{tabular}{|c|c|c|c|}
\hline & \multicolumn{3}{|l|}{ Dependent variable } \\
\hline & \multicolumn{3}{|c|}{ Average absolute distance } \\
\hline & (1) & (2) & (3) \\
\hline$\widehat{\beta}_{1}:$ Intercept & $0.264(0.0160)^{* * *}$ & $0.264(0.0152)^{* * *}$ & $0.264(0.0144)^{* * *}$ \\
\hline \multicolumn{4}{|l|}{$\begin{array}{l}\text { Convergence targets by } \\
\text { treatment }\end{array}$} \\
\hline$\widehat{\beta}_{21}:$ Discrete time & $0.140(0.0004)^{* * *}$ & $0.140(0.0022)^{* * *}$ & $0.140(0.0003)^{* * *}$ \\
\hline$\widehat{\beta}_{22}:$ Continuous slow & $0.063(0.0068)^{* * *}$ & $0.063(0.0047)^{* * *}$ & $0.063(0.0052)^{* * *}$ \\
\hline$\widehat{\beta}_{23}:$ Continuous instant & $0.048(0.0080)^{* * *}$ & $0.048(0.0036)^{* * *}$ & $0.048(0.0042)^{* * *}$ \\
\hline \multicolumn{4}{|l|}{$\begin{array}{l}\text { Average group normalized } \\
\text { robot score }\end{array}$} \\
\hline$\widehat{\rho}$ & & $-0.035(0.0053)^{* * *}$ & \\
\hline$\widehat{\rho}_{D S}:$ Discrete time & & & $-0.005(0.0005)^{* * *}$ \\
\hline$\widehat{\rho}_{C S}:$ Continuous slow & & & $-0.018(0.0091)^{*}$ \\
\hline$\widehat{\rho}_{C I}:$ Continuous instant & & & $-0.045(0.0058)^{* * *}$ \\
\hline Adjusted $R^{2}$ & 0.616 & 0.6742 & 0.6835 \\
\hline Residual std. error & 0.052 & 0.04789 & 0.0472 \\
\hline F statistic & $\begin{array}{c}26,297(d f=4 \\
65,488)^{* * *}\end{array}$ & $\begin{array}{c}27,110(d f=5 \\
65,487)^{* * *}\end{array}$ & $\begin{array}{c}20,210(d f=7 \\
65,485)^{* * *}\end{array}$ \\
\hline Number of observations: & 65,492 & 65,492 & 65,492 \\
\hline
\end{tabular}

Data are from periods 7 through 12 of all games involving four human players. Standard errors in parentheses; $* * * *<0.01$ and $* p<0.1$ 
$\widehat{\beta}_{1}=0.264$ of the origin parameter, but the main point is that the estimated asymptotic distance $\widehat{\beta}_{21}$ is not much below the random benchmark of 0.17 .

Result 2 Continuous time treatments exhibit convergence toward the pure Nash equilibrium.

Support for Result 2 The estimated asymptotic distances from NE in the Slow and Instant treatments are $\widehat{\beta}_{22}=0.063$ and $\widehat{\beta}_{23}=0.048$ respectively. These are statistically different from zero, but "economically" speaking they are much closer to zero than to the random benchmark 0.17 . We find significant differences between the discrete and each continuous time treatment convergence targets estimates $\left(H_{0}\right.$ : $\beta_{21} \neq \beta_{22}, \beta_{21} \neq \beta_{23}$ at $p<0.001$ in pairwise test correcting first order autocorrelation, and clustering errors at the silo level). We conclude that behavior moves decisively towards (but not all the way to) the Nash equilibrium in these treatments.

Result 3 Convergence toward NE is better in the continuous Instant treatment than in the continuous Slow treatment.

Support for Result 3 The coefficient estimate $\widehat{\beta}_{23}=0.048$ for Instant is less than the estimate $\widehat{\beta}_{22}=0.063$ for Slow, though these estimates are not statistically different. A more nuanced picture can be obtained by comparing the empirical distributions of average absolute distance from NE across treatments, as in Fig. 6. One can see that the Discrete treatment has a vastly different distribution compared to the two continuous treatments, and that Instant has a much larger fraction of tiny distances (on or very near NE) than Slow. The Figure also shows that the distributions for the continuous time treatments are close for distances exceeding about 0.1 , i.e., the upper tails are similar.

Table 3 lists the percent of AvgAbsDist observations for which it is implied that subjects are 1 and $5 \%$ away from the pure Nash equilibrium, i.e. the percent of AvgAbsDist observations less than 0.0025 and 0.0125 respectively. Regarding "within $5 \%$ " as close, we see that in $26 \%$ of observations players in continuous Instant treatments are close to the equilibrium configuration, compared to just $6 \%$ of observations in the continuous Slow treatment and much less than 1 percent of Discrete time observations. Regarding "within 1 percent" as close, the ratios of the percent of close-to-NE observations swings even more sharply towards the Instant treatment although, of course, the percentages are smaller than for the less stringent criterion for closeness.

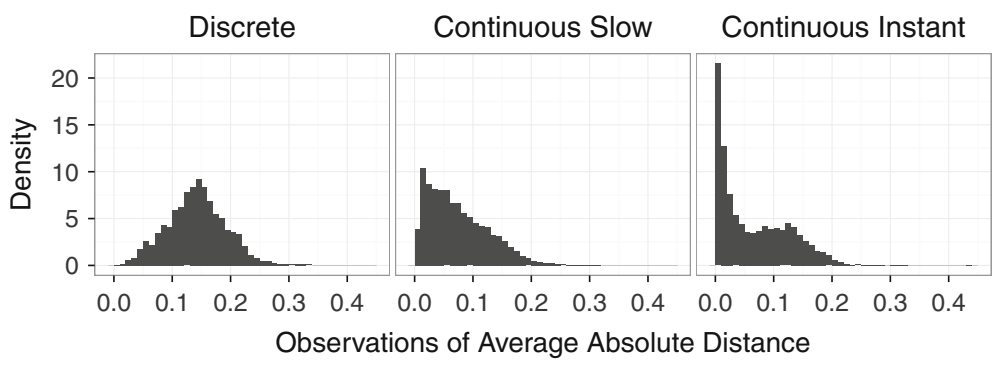

Fig. 6 Histograms of Average Absolute Distance 
Table 3 Attainment of near-equilibrium location formations by treatment

\begin{tabular}{lllc}
\hline & Discrete $(\%)$ & Continuous slow $(\%)$ & Continuous instant $(\%)$ \\
\hline Periods 1-3 & $0.0(0.0)$ & $0.01(2.53)$ & $0.3(8.76)$ \\
Periods 4-6 & $0.0(0.0)$ & $0.02(4.33)$ & $0.41(22.08)$ \\
Periods 5-9 & $0.0(0.19)$ & $0.01(6.59)$ & $8.5(33.51)$ \\
Periods 10-12 & $0.0(0.0)$ & $0.74(12.05)$ & $6.28(39.06)$ \\
Overall & $0.0(0.05)$ & $0.20(6.38)$ & $3.87(25.86)$
\end{tabular}

Entries are the fraction of observations within $1 \%$ of pure NE (AvgAbsDist $\leq 0.0025)$, and within within $5 \%$ of pure NE in parentheses (AvgAbsDist $\leq 0.0125$ ). Observations consist of subject location configurations sampled ten times a second in continuous time treatments, and each subperiod in the discrete time treatment

Result 4 In the Instant treatment, groups of players that perform well against robot agents tend to converge more closely towards the pure NE.

Support for Result 4 We compute the average group normalized robot score, $\rho$, for each four-human-players group. ${ }^{3}$ Column (2) of Table 2 shows that including this explanatory variable in Eq. 4 yields a very significant coefficient estimate of 0.035 . That is, a standard deviation increase in a group's average ability against robots is associated with a $3.5 \%$ smaller deviation from NE, holding treatment effects constant.

Column (3) of Table 2 shows that the strength of this effect varies by treatment. The interaction $\rho_{D S}=-0.005$ is statistically but not economically significant, while the interaction $\rho_{C S}=-0.018$ is three times larger but only marginally significant. The very significant Instant treatment interaction, $\rho_{C I}=-0.045$ indicates that how well the four-player group did in our automated agent periods is particularly important in achieving convergence to the static NE in the continuous Instant setting.

\section{Discussion}

Our interest in Hotelling location games led us to focus on the $n=4$ player case. Our laboratory data replicate the negative result of Huck et al. (2002) that behavior fails to converge in the Discrete time treatment. Thus we reject the Testable Hypothesis 1 for this treatment. More importantly, we have identified (as far as we know, for the first time) conditions that lead to good NE convergence. In both continuous time treatments, Slow and Instant, we get convergence to a neighborhood of (but not precisely to) NE both within a typical period and across periods. The neighborhoods are tighter under Instant, as confirmed by parametric tests in the

\footnotetext{
${ }^{3}$ Details are as follows. For each player $i$, we compute the total payoffs $R_{i}$ against robots, and find the average $\bar{R}_{\tau}$ and standard deviation $\sigma_{\tau}$ across all players in each treatment $\tau$. The normalized individual score $\rho_{i}=\frac{R_{i}-\bar{R}_{\tau}}{\sigma_{\tau}}$ is comparable across individuals and treatments. These individual level scores are averaged across the four players matched in a given group, providing a indication of group's overall ability. Group-level $\rho$ aligns with group-period level AvgAbsDist time series.
} 
tradition of Noussair et al. (1995). This supports Testable Hypothesis 1 for these treatments, and also supports Testable Hypothesis 2.

We believe that our results have a practical implication: Hotelling location models now seem more relevant to understanding behavior in the wider world than might previously have been supposed. In particular, in terms of product positioning in a space of features or perceived characteristics, our Instant and Slow treatments capture important aspects of competition in the 21st century. In some situations (e.g., introducing new products) firms are able to place their product anywhere in the spectrum, while in other situations (e.g., repositioning) product development and marketing can only gradually adjust the way consumers perceive their product. Our results suggest that static Nash equilibrium may have predictive power in both situations.

There also may be theoretical implications, regarding the dynamic foundations of static Nash equilibrium in general and not just for the particular spatial model we investigate. Many early game theorists, including John Nash, offered intuitive dynamic arguments that later generations of theorists formalized as learning in games or evolutionary games; for a now-classic summary see Fudenberg and Levine (1998). Our work speaks to a followup question: when (and how) do continuous time dynamics alter convergence to static NE relative to discrete time dynamics? Friedman and Oprea (2012) and Bigoni et al. (2015) find that continuous time dynamics dramatically delay convergence to the inefficient stage game NE in prisoner's dilemma games, i.e., they support cooperation in the finite horizon repeated game. Dorsey (1992) and Oprea et al. (2014) find that, by itself, continuous time is insufficient to reliably delay convergence to the inefficient NE in four player voluntary contribution public goods games, perhaps due to coordination issues. Deck and Nikiforakis (2012) study a challenging pure coordination game: minimum effort or weakest link played on a circle network. Their results suggest that strategic uncertainty impedes convergence to the more efficient static NE. Our own results, including the simulation exercises, provide additional evidence.

Definitive answers to the followup question await further work, but theorists pondering that question may wish to distinguish the impact of asynchronous responses from rapid responses. Coordinating on a static NE (which emphasizes unilateral best response) may be aided mainly by asynchronous response opportunities, while coordinating on an efficient profile (not an issue in a Hotelling location model) may be aided mainly by rapid responses.

Several new avenues of laboratory research now come into focus. First, we note that unexplained discrepancies remain between our Discrete time results and those of Huck et al. (2002). They obtain sharper modes than we do, including the contraNE mode at the center location.

A broader avenue is to apply the continuous time treatments to more general Hotelling models, including the no-edge case (the circle), joint decisions of price and location, and different numbers of players. Another broad avenue is to separate the impact of continuous time per se from the impact of asynchronous choice. Our agent-based simulations suggest that taking turns in discrete time suffices to achieve Nash (or near-Nash) equilibrium behavior, but we do not yet know whether humans will agree. 


\section{References}

Bigoni, M., Casari, M., Skrzypacz, A., \& Spagnolo, G. (2015). Time horizon and cooperation in continuous time. Econometrica (forthcoming).

Kruse, J. B., \& Schenk, D. J. (2000). Location, cooperation and communication: An experimental examination. International Journal of Industrial Organization, 18(1), 59-80.

Brown-Kruse, Jamie, Cronshaw, M. B., \& Schenk, D. J. (1993). Theory and experiments on spatial competition. Economic Inquiry, 31(1), 139-165.

Collins, R., \& Sherstyuk, K. (2000). Spatial competition with three firms: an experimental study. Economic Inquiry, 38(1), 73-94.

Deck, C., \& Nikiforakis, N. (2012). Perfect and imperfect real-time monitoring in a minimum-effort game. Experimental Economics, 15(1), 71-88.

Dorsey, R. E. (1992). The voluntary contributions mechanism with real time revisions. Public Choice, 73(3), 261-282.

Downs, A. (1957). An economic theory of political action in a democracy. The Journal of Political Economy, 135-150.

Eaton, B. C., \& Lipsey, R. G. (1975). The principle of minimum differentiation reconsidered: some new developments in the theory of spatial competition. The Review of Economic Studies, 27-49.

Friedman, D., \& Oprea, R. (2012). A continuous dilemma. The American Economic Review, 102(1), 337-363.

Fudenberg, D., \& Levine, D. (1998). Learning in games. European economic review, 42(3), 631-639.

Greiner, B. (2004). An online recruitment system for economic experiments.

Hotelling, H. (1929). Stability in competition. The Economic Journal, 39(153), 41-57.

Huck, S., Müller, W., \& Vriend, N. J. (2002). The east end, the west end and king's cross: on clustering in the four-player hotelling game. Economic Inquiry, 40(2), 231-240.

Netz, J. S., \& Taylor, B. A. (2002). Maximum or minimum differentiation? location patterns of retail outlets. Review of Economics and Statistics, 84(1), 162-175.

Newey, W. K., \& West, K. D. (1986). A simple, positive semi-definite, heteroskedasticity and autocorrelationconsistent covariance matrix. National Bureau of Economic Research Cambridge, Mass., USA.

Noussair, C. N, Plott, C. R., \& Riezman, R. G. (1995). An experimental investigation of the patterns of international trade. The American Economic Review, 462-491.

Oprea, R., Charness, G., \& Friedman, D. (2014). Continuous time and communication in a public-goods experiment. Journal of Economic Behavior \& Organization, 108, 212-223.

Pettit, J., Friedman, D., Kephart, C., \& Oprea, R. (2013). Software for continuous game experiments. Experimental Economics, 1-18.

Shaked, A. (1982). Existence and computation of mixed strategy nash equilibrium for 3-firms location problem. The Journal of Industrial Economics, 93-96. 